\title{
The Effects of Resistance and Endurance Training on the Liver Tissue FNDC5 mRNA Gene Expression in Male Rats
}

\author{
${ }^{1}$ Amir Rashidlamir, ${ }^{2}$ Mohammad Hoseinzadeh, ${ }^{3}$ Leili Zeiaddini Dashtkhaki
}

\begin{abstract}
${ }^{1}$ Department of Exercise Physiology, Faculty of Sport Sciences, Ferdowsi University of Mashhad, Mashhad, Iran. ${ }^{2}$ Department of Physical Education, Farhangian University, Tehran, Iran. ${ }^{3}$ Department of Physical Education, Kerman Branch, Islamic Azad University, Kerman, Iran.
\end{abstract}

\begin{abstract}
Background. The accumulation of excess triglyceride in the liver and a decrease in brown adipose tissue is related to the fatty liver disease. Regular physical activity can take part in regulating fat oxidation and inhibiting fat accumulation by creating and releasing some myokines. Nevertheless, the effectiveness of each training method in this regard is still not certain. Objective(s). This study aims at comparing the effects of resistance and endurance training on FNDC5 (the precursor of irisin myokine) gene expression in male rat liver tissues.. Methods. Fifteen Wistar male rats (aged 10-12 weeks with an average weight of $331.8 \pm 63.09$ gr) were used. After being taken to the animals' laboratory, the subjects were randomly assigned to three equal groups viz: control $(\mathrm{N}=5)$, endurance $(\mathrm{N}=5)$ and resistance $(\mathrm{N}=5)$. For the purpose of tissue sampling, all these subjects were anesthetized 72 hours after the experiment had been completed. The liver tissue was immediately removed and then quickly frozen in liquid nitrogen and kept at a temperature of -80o C until RNA extraction. FNDC5 relative gene expression was identified by Real-time PCR method. Results. Data analysis revealed a significant difference in FNDC5 gene expression among the groups $(\mathrm{P}=0.008$, Chi Square=31.791). No significant difference was observed between the resistance training and endurance training groups in FNDC5 gene expression, though $(\mathrm{P}=0.959)$. Conclusion. It can be established that both resistance and endurance training can similarly affect the production and secretion of exerciseinduced myokines, including FNDC5 irisin, which can in turn protect against obesity and its impacts on the liver tissue by stimulating the process of browning of white fat.
\end{abstract}

KEY WORDS: FNDC5, Liver, Resistance Training, Endurance Training.

\section{INTRODUCTION}

Mammals provide the energy needed for metabolic functions by storing several macromolecules, such as carbohydrates, proteins and fats as fuel. Organs involved in fuel storage, such as the liver, the skeletal muscle and adipose tissues all communicate with each other and determine fuel selection and delivery under the strict control of neuroendocrine system (1). The liver stores energy as glycogen and stores digested triglycerides in the form of fatty acids (2). On the contrary, the accumulation of excessive triglyceride in the liver can result in hepatic steatosis (3). Adiposity can be seen either in the form of white adipose tissue or

*. Corresponding Author:

Amir Rashidlamir

E-mail: rashidlamir@um.ac.ir 
brown adipose tissue. White adipose tissue, the physiological area of triglyceride storage, accounts for a major part of fat in humans. Conversely, brown adipose tissue is the only source of energy expenditure and thermogenesis without trembling in small mammals and human infants (4). In fact, the main function of the brown adipose tissue is energy expenditure, which mostly takes place in an increased expression of the uncoupling protein 1. This protein triggers uncoupling in mitochondrial respiration, promoting energy loss in the form of heat. On the contrary, brown fat appears to be protective for metabolic diseases and its mass increases according to cold exposure or physical activity (5).

Physical exercise stimulates the release of myokines which are soluble factors released by skeletal muscle in response to muscle fiber contraction showing auto-, para-, and endocrine functions. These myokines have been shown to act as messengers among skeletal muscle, liver, adipose tissue, heart, brain, and blood vessels (5). A large list of molecules have been recently considered as myokines, such as irisin, fibroblast growth factor-21 (FGF21), interleukin-6 (IL-6), IL-7, IL-8, IL-15, leukemia inhibitory factor (LIF), myonectin, myostatin, vascular endothelial growth factor (VEGF), brain-derived neurotrophic factor (BDNF) and follistatin-like 1 (FSTL1) (5-7).

Irisin, a 112 -amino acid protein $(12587 \mathrm{kDa})$, is a thermogenic protein that triggers energy consumption by converting white adipose tissue to brown adipose tissue. Irisin is a cleavage product that is produced when fibronectin domain containing 5 (FNDC5) is cleaved (8). It was initially discovered and characterized by two independent groups in 2002. In 2011 FNDC5 was introduced by Boström and colleagues as the precursor of irisin (9).The expression of FNDC5 has been traced in the heart, brain, ovary, testicles, kidneys, rectum, epiglottis, oviduct, adrenal, tonsil, esophagus, vena cava, hypophysis, retina, thyroid, small intestine, spinal cord, skeletal muscle and the liver, which has also attracted lots of attention $(8,10,11)$. One of the target proteins of PPAR $\gamma$ coactivator-1 $\alpha(\mathrm{PGC} 1 \alpha)$ is FNDC5 (6). PPAR $\gamma$ are nucleonic receptors that function to modify the expression of the genes involved in lipid and glucose metabolism and have also been referred to as atherosclerosis inhibitors (12). Conversely, recent groundbreaking experiments in mice indicate that the transcriptional coactivator, PGC-1 $\alpha$, which promotes biogenesis of mitochondria, drives the expression of FNDC5, which in turn leads to increased brown adipose tissue, increased $\mathrm{Vo}_{2}$, insulin sensitivity, and glucose tolerance (13).

Physical exercise is one of the major nonpharmacological technique for controlling frequently encountered metabolic disorders; yet the mechanisms which enact exercise-induced effects remain elusive. It was suggested that during muscular exercise, peroxisome proliferator-activated receptor-gamma (PPAR$\gamma)$ coactivator-1 $\alpha$ (PGC1 $\alpha)$ expression is increased in the muscle [3]. This induces the expression of fibronectin type-III domaincontaining 5 (FNDC5), which is then cleaved and secreted as irisin (14). This can trigger energy hemostasis and metabolism, improve obesity and glucose intolerance, which is why it is known as a beneficial factor in recovering from disease (2). Physical activity is the basis of change in lifestyle which is of great importance for patients with non-alcoholic fatty liver disease (15). A recent research in this regard made it evident that physical activity can result in the lowering of liver osteotasis. The co-activator of this lowering is unknown, but it was recently shown that the levels of plasma irisin is inversely related to the triglyceride content of the liver and it was suggested that irisin was involved (16).

In fact, irisin is known as an exercise-induced myokine (17) and several studies have documented the relationship between FNDC5/irisin expression and intensity, duration and type of exercise $(11,18,19)$. The expression that irisin is an exercise-induced myokine dependent on PGC-1a is based on the research carried out by Boström et al. (2012), who managed to demonstrate that the levels of FNDC5 mRNA in mice's skeletal muscle had an increase of about 2.8 times after 3 weeks of optional training on the treadmill. Furthermore, they reported that FNDC5 expression cooccurred with an increase in PGC-1a mRNA (20).

In spite of extensive examinations of the metabolic functions of this novel protein, few 
studies have examined the mechanisms by which acute exercise/muscle contractions increase FNDC5 expression (21). Therefore, it remains unclear which kind of exercise protocol, intensity or duration, if any, would be optimal to increase the circulation of serum irisin levels in the general population (22). Specifically, the comparison of different exercises and their correspondent influence on FNDC5 expression has, thus far, led to no reliable results, particularly in terms of the liver as a major glucose-regulating organ and adipocyte metabolism. All the same, the current study seeks to compare the effects of two protocols of endurance training and resistance training on the liver FNDC5 gene expression in male rats.

\section{MATERIALS AND METHODS}

Animals. This is an experimental study in which 15 male rats (Wistar race, 10-12 weeks old with an average weight of $331.8 \pm 63.09$ gr) were equally allocated into three groups of control, aerobic exercise and resistance (5 in each group). These rats were kept in controlled light conditions ( 12 hours of dark after 12 hours of light), temperature ( $22 \pm 1$ degrees centigrade) and humidity (50\%) in transparent polycarbonate cages meant for rodents and they had free access to food and water. All animals received humane care according to the criteria outlined in the "Guide for the Care and Use of Laboratory Animals" prepared by the National Academy of Sciences and published by the National Institutes of Health (NIH publication 86-23 revised 1985).

Exercise Training Protocol. Before the commencement of the training protocol, the subjects spent a week getting acquainted with the procedure. The familiarization process for the aerobic group included 5 sessions of walking on the treadmill at a speed of 15-20 meters per minute and a zero slope which lasted for 20-40 minutes. For the resistance group, it comprised 5 sessions of climbing a one-meter-high ladder with a slope of 60-80 degrees. This is followed by the attachment of the clipper and the connected weight to the rat's tail.

Subsequently, the endurance group performed the aerobic exercise, which was made up of 8 weeks' (5 days a week) running at a speed of $20 \mathrm{~m} / \mathrm{min}$ for 60 minutes in each session. The first 10 minutes was allocated for warmup and the final 10 minutes to cool down at a speed of $15 \mathrm{~m} / \mathrm{min}$ (23).

The resistance training was carried out in 3 sessions per week for 8 weeks. During the first week, the weight connected to each subject's tail equaled $50 \%$ of the rat's bodyweight, and it had a $10 \%$ increase per week. The training was done on a one-meter-high ladder so that a weight was attached to the clipper on the rat's tail, and the animal had to climb up the ladder. This was done in three rounds and repeated five times. The rounds' intervals were 3 minutes and the interval for repetitions was 1 minute. This training method was adapted from reliable sources (24, 25). The control group performed no exercise during this period.

Tissue Biopsies. 72 hours after the final exercise session, the animals were anaesthetized in the specialized sampling site (sterile space) with a combination of ketamine (30-50 $\mathrm{mg}$ for each $\mathrm{kg}$ of weight) and xylazine (3-5 $\mathrm{mg}$ for each $\mathrm{kg}$ of weight) (25). When anesthesia was ensured via not backing of the leg on touch, a rupture was made on the skin in the abdominal region and when the liver appeared, tissue sampling was conducted. The sample was then immediately cleansed using cooled physiological serum and inserted in a microtube and then frozen in liquid nitrogen. Liver samples were then transferred to a freezer at a temperature of $-80{ }^{\circ} \mathrm{C}$.

RNA, cDNA Synthesis and Real-time PCR. In order for mRNA to be taken, the tissue was washed twice with warm PBS and then placed in 1.5 microliter microtubes. $1 \mathrm{ml}$ of Tripure was added to each tube. The tissue was pipetted severally for the cells to be perfectly lubricious. The homogenized samples were kept at room temperature for 5 minutes. Then 200 microliters of chloroform was added to each tube and they were thoroughly shaken. The tubes were incubated for 15 minutes and then centrifuged at a speed of $12000 \mathrm{~g}$ at a temperature of $4^{\circ} \mathrm{C}$ for 15 minutes. The topmost phase was smoothly transferred to another sterile microtube and 500 microliters of isopropanol was added and reversed several times. The samples were incubated at room temperature for 10 minutes. The samples were centrifuged at a speed of $12000 \mathrm{~g}$ at a temperature of $4^{\circ} \mathrm{C}$ for 10 
minutes. The topmost solution was discarded and $1 \mathrm{ml}$ of ethanol $75 \%$ was added. The RNA plate was washed in ethanol by vortexing and the samples were centrifuged at a speed of $7500 \mathrm{~g}$ for 5 minutes. The topmost solution was discarded and was allowed for the RNA deposit to become semi-arid. This deposit was dissolved in 20 microliters of DEPC water. This solution was incubated at a temperature of $55^{\circ} \mathrm{C}$ for 15 minutes and immediately subjected to $-80{ }^{\circ} \mathrm{C}$. Subsequently, by the means of a cDNA synthesis kit (BIONEER, Korea), cDNA was synthesized from the RNA. The nucleotide primer sequences of FNDC5 gene (25) and the reference gene (26) are given in Table 1. Real Time PCR was run to measure the level of gene expression and was programmed with an initial step of 5 mins at $95^{\circ} \mathrm{C}$ ( 1 cycle), followed by 40 cycles of 15 seconds at $95^{\circ} \mathrm{C}$ and 30 seconds at $60^{\circ}$. The parameter for melting curve program was $72^{\circ} \mathrm{C}$ for 30 seconds.

Table 1. Oligonucleotide Primer Sequences and Real-time PCR Amplification Parameters Temperature

\begin{tabular}{cccc}
\hline Gene & Forward and reverse & Forward and reverse primer sequences & Annealing temperature \\
\hline & $\mathrm{F}$ & 5 -GTCTCCCACCACCATCTT-3 & $\mathbf{6 3}$ \\
FNDC5 & $\mathrm{R}$ & 5-TCTGTCTCTGAGTGTAGCCTTAGC-3 & $\mathbf{6 3}$ \\
\hline & $\mathrm{F}$ & 5'-GTGCCAGCCTCGTCTCATAG & $\mathbf{6 0}$ \\
\hline
\end{tabular}

After the completion of the reaction, the threshold cycle $(\mathrm{Ct})$ was calculated for each sample. The proportion of the gene $\mathrm{Ct}$ to that of the housekeeping gene gave the level of gene expression via the method $2^{-\Delta \Delta C t}$ by means of the following equation (27): the $\mathrm{Ct}$ for each sample's housekeeping gene is subtracted from the $\mathrm{Ct}$ for that very sample's gene.

$(\Delta \mathrm{Ct}=\mathrm{Ct}$ Target- $\mathrm{Ct}$ Housekeeping)

As for the next step, $\Delta \mathrm{Ct}$ for each sample is subtracted from that of the sample with which it will be compared with, and the negative result is applied as the power of two so that the relevant gene expression is achieved.

( $\Delta \Delta \mathrm{Ct}=\Delta \mathrm{Ct}$ Target $-\Delta \mathrm{Ct}$ Reference)

$\mathrm{E}=2^{-\Delta \Delta \mathrm{Ct}}$

Statistical Analysis. Shapiro Wilk test did not yield a normal distribution of data. Thus, to make a comparison of the mean of the groups, Kruskal Wallis non-parametric test was run. The level of significance was set to $\mathrm{P} \leq 0.05$. The data was analyzed using SPSS 16.

\section{RESULTS}

Results of a Shapiro Wilk test revealed no normal distribution of data and Kruskal Wallis test demonstrated a significant difference in FNDC5 gene expression among the groups $(\mathrm{P}=0.008$ and Chi-Square $=9.27)$. A subsequent LSD test revealed a significantly higher
FNDC5 gene expression of the liver both in the endurance and resistance groups than in the control group $(\mathrm{P}<0.033$ and $\mathrm{P}<0.030$, respectively) (Figure 1). However, no significant difference was found in FNDC5 gene expression between the aerobic exercise group and the resistance training group $(\mathrm{P}=0.959)$.

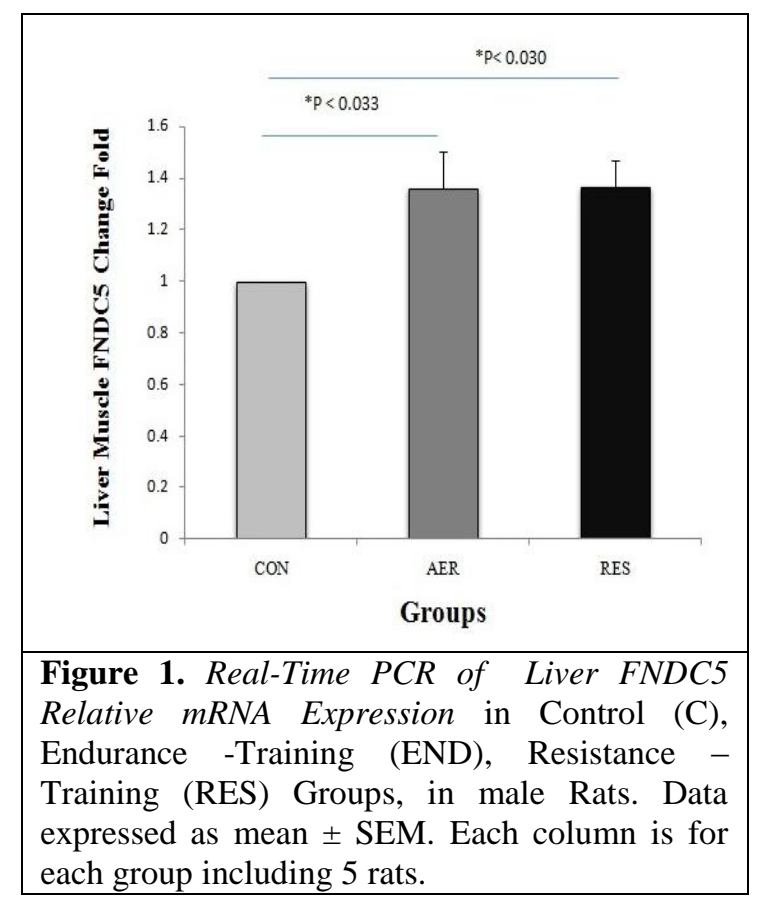




\section{DISCUSSION}

The findings of this study concerning the level of liver NDC5 gene expression revealed a significant increase of that gene in response to endurance and resistance training.

Recent studies showed that FNDC5 was also expressed in other tissues such as heart, adipose tissue and liver, which indicates supplementary functions of this hormone (3). The liver stores energy as glycogen and stores digested triglycerides in the form of fatty acids (2).

Generally, a small amount of the fat and carbohydrates coming from the meals is stored in the liver. Lipolysis (found in the adipose) and lypogenesis in the liver also take part in the liver fat mass storing, but in return, the process of fat disposal maintains this mass at a low level. Nevertheless, if the adipose tissue is unable to store additional free fatty acids, the liver will be transformed into a place where fat can be stored and a lot of fat will be accumulated in the liver (28). Furthermore, changes in the amount of fat produced and fat removal through the oxidation of fatty acids or secretion of lipoproteins rich in triglyceride from the liver take part in this imbalance $(29,30)$. In cases such as obesity, excessive nutrition intake and resistance to insulin, the balance between adipose collection and fat removal is disturbed, which can result in NAFLD (29). The collection of excess fat in the adipose tissue and the liver causes inflation and metabolic stress, which brings about NAFLD (31). NAFLD can be enhanced through weight reduction and exercise, without medical therapy (2). FNDC5 mRNA/irisin is produced not only in skeletal muscles of humans and animals, but also in other tissues including the liver, though to a limited extent (32-35), which is involved in energy homeostasis and the metabolism of the body; hence, a connector of the skeletal muscle and the other tissues $(32,36)$. Particularly with respect to the liver, it appears that irisin is produced by the liver cells, Kupfer cells and endothelial sinusoids $(34,35)$. The function of the liver-derived irisin is not yet known. It may play a local role through the autocrine and paracrine routes and an endocrine role through the circulation system. The irisin in the liver may be related to fat and carbohydrate metabolism and also resistance to liver insulin (37). People with NAFLD often have an iatrogenic lipid profile identifying them with high LDL and low HDL (38). In other words, an important aspect of the disease in such people is an increase in intraliver triglyceride resources with/without inflation or fibrosis. Intra-liver triglyceride is strongly connected with BMI, waist circumference, high blood pressure, level of insulin and trans aminazes (ALT, AST) (40). HDL cholesterol acts against arthrosclerosis through its antiinflation and control function on the process of cholesterol transfer (40). In accordance with previous research, the indirect correlation between the level of intra-liver triglyceride and irisin and the direct correlation between irisin and HDL can reinforce irisin's protective potential particularly in people with such chronic diseases as NAFLD, which generate cardiovascular risks (41). Irisin can directly or indirectly prevent the accumulation of triglyceride in the liver. With this regard, irisin may manipulate PPAR- $a$, which is an instrumental regulator in fat metabolism that modifies fat oxidation through a thermogenesis mechanism (42). Additionally, PPAR-a regulates fibroblast 21 growth factor (FGF21), which can ameliorate liver osteatosis and insulin sensitivity (43). Therefore, irisin can regulate the amount of intra-liver triglyceride through FGF21. Furthermore, a decline in the plasma irisin causes a rise in ALT and AST, signifying that irisin can function as a protective against liver steatosis (16).

The beneficial effects of a balanced diet along with physical activity are welldocumented in that they comprise the basis of non-pharmacological treatment of cardiovascular and metabolic diseases. Apart from enhancing resistance and strength, physical activity promotes energy expenditure, which reduces the adipose tissue and has protective power against chronic diseases such as obesity and type 2 diabetes. However, the mechanisms leading to these effects are not definitely known (5).

Eight weeks of endurance training and resistance training demonstrated that both protocols can similarly manipulate the expression of the liver FNDC5 mRNA, which was consistent with the previous research (13, 17, 22, 25). But it is inconsistent with Fain et al. (2012), who reported that 16-20 weeks physical training caused no significant effects on FNDC5, 
subcutaneous, deltoid, triceps and heart muscle of pigs (44). These researchers suggested that the rise in plasma irisin levels, as opposed to the absence of such an increase in FNDC5 gene expression in training pigs could be due to the fact that physical activity might affect postmodelling phases in irisin expression (including the breakdown of FNDC5 to produce irisin). However, this requires closer attention. Among the possible sources of inconsistency between this study and that conducted by Fine et al. (2013) is the fact that different subject types were employed. The latter used pigs as their subjects, whereas the former made use of rats. According to previous research, uncoupling protein 1 (UCP-1) is non-functional in pigs (4546). Since this non-functionality of UCP-1 appears not to be harmful in pigs, it could be said that irisin influences energy metabolism in pigs including signaling which is different from that in rats. Another source could be the fact that in this research, it was the liver tissue which was studied, while in Fine et al. (2013) it was the triceps, dally and the heart. Other factors were the sampling time (they took their sample 24 hours after the last training session, while in this study, sampling was done after 72 hours) and the type of training (they used increasing aerobic exercise).

Our research was also inconsistent with that of Pekkala et al. (2013), who found no differences in FNDC5 and serum irisin levels after long-time acute aerobic exercise or after a combination of aerobic and resistance training. They just managed to notice a rise in FNDC5 expression in young men, following a single session of resistance training. They also demonstrated that alterations in PGC-1a levels were not associated with FNDC5. Finally, they concluded that there is little evidence to confirm any definitive link between exercise and FNDC5 expression and irisin release in humans, and further research ought to deal with the regulatory mechanisms (22). In order to explain their findings, Pekkala et al. (2013) argued that too much variety in FNDC5 and irisin intrapersonal and interpersonal response to different types of training makes it obvious that physical activity cannot increase FNDC5 mRNA in the skeletal muscles or the circulating irisin in all individuals. They also stated that the sampling time may also affect the results. Another factor contributing to this inconsistency could be their subject type (their subjects were humans) and the sampled tissue (the skeletal muscle). The sampling time and training types were also different. All these should be noted as likely sources of disagreement.

Irisin is a myokine whose levels appear to increase during physical exercise leading to the generation of heat and a possible protective effect on metabolic disorders. Molecular mechanisms underlying irisin, combined with the increase of brown fat, may unravel the basis of physical exercise benefits on different conditions (5). Irisin, a hormone proteolytically processed from FNDC5, has been reported to induce the browning of subcutaneous adipocytes by increasing the level of uncoupling protein 1(Ucp1) (3). It is well-documented that the brown adipose tissue is indirectly proportional to BMI and resists obesity by controlling energy expenditure (47). Insufficient activity of this tissue can play a role in collective pathogenesis of intra-liver triglyceride. In other words, a decrease in brown adipose tissue can increase the risk of NAFLD (48).

FNDC5 is one of the target proteins of PPAR $\gamma$ and PGC-1a (6). PPAR $\gamma$ proteins are nucleonic receptors that function to modify the expression of the genes involved in lipid and glucose metabolism and have also been known as inhibitors of atherosclerosis (12). PPAR $\gamma$ and PGC-1a are capable of regulating several genes in response to nutritional and physiological signals. Over expression of these genes is associated with weight loss and a decrease in the levels of inflammatory muscular markers and stress oxidative. Boström et al. (2012) examined mouse samples over a long-term program and observed that excess PGC-1a is correlated with UCP1 upregulation in some subcutaneous cells. One of the possibly involved factors was FNDC5, whose transmembrane increase was outstandingly observable. In that study, a 15-fold increase in FNDC5 expression was observed, 4fold for irisin and 15-fold for UCP1. The observations also included increase in oxygen consumption, weight loss, better glucose tolerance and reduced insulin secretion (20). Another aspect that was investigated by Boström et al. (20) was how UCP1 is upregulated, being 
the most probable mechanism that increased the expression of PPAR $\alpha$. PPAR $\alpha$ is a member of the family of PPAR ligand-activated receptors, which play roles in the control of lipid and glucose metabolism. Over-expression of FNDC5 increases the expression of PPAR mRNAs in white adipocytes up to 3-fold. Furthermore, the pharmacological inhibition of PPAR $\alpha$ stops fat browning, signifying a possible role of PPAR in mediating the effects of FNDC5 (5). This relationship between irisin and PPAR $\alpha$ signaling is very interesting, given that this signaling pathway is known to play a vital role in hepatic $\beta$-oxidation. Irisin may modulate the PPAR $\alpha$ signaling pathway, a key regulator of lipid metabolism that coordinates fat oxidation through a thermogenesis mechanism (5). Previous studies have reported an increase in PPAR $\alpha$ following physical training $(26,49,50)$. It seems practicable that endurance training and resistance training are beneficial in adipose metabolism by elevating the expression of FNDC5 and the subsequent increase in PPAR $\alpha$ expression stimulation. However, PPAR gene expression was not measured in the present study.

One possible scenario concerning the effect of long-term resistance training on body composition is that these exercises create signals which activate PGC-1a in the skeletal muscle and this, in turn, induces the expression of FNDC5. Irisin operates on white adipose tissue and elevates UCP1, which signals an increased thermogenesis and energy expenditure $(2,25)$. However, as there is no established relationship between FNDC5 and PGC-1a alterations, some studies have suggested the possibility that there may be other factor(s) involved in the regulation of FNDC5 expression, with PGC-1a being the only major regulator of energy metabolism (21).

These contradictory findings allow for an alternative path for the regulation of FNDC5 expression. Mo et al. (2015) showed that a novel path regulates FNDC5/irisin expression through $\mathrm{CAR}$ in the mouse liver. It is revealed that FNDC5/irisin is the direct target of CAR transcription. CAR (constitutive androstane receptor) is a member of nucleonic receptors that induces FNDC5 expression in the mouse liver and increases serum irisin. In other words, CAR is a xenobiotic sensor that controls the expression of genes involved in the metabolism of exogenous molecules and endogenous compounds such as bile acids, bilirubin and thyroid hormones. Activation of CAR inhibits hepatic steatosis by repression of lipogenesis and increases fatty acid B-oxidation. Activation of CAR also inhibits diet- and genetic-induced obesity and insulin resistance by reducing the expression of glucose-6-phosphatase (G6p) and phosphoenol pyruvate carboxykinase (Pepck), two key enzymes in gluconeogenesis (3).

Mo et al. (2015) elucidated the involvement of this pathway in the regulation of FNDC5/irisin expression and also determined the physiological role that this hormone plays in glucose and adipose hemostasis. As a result, they explained the role of irisin in adipose and hepatic glucose metabolism, providing a potential treatment target for metabolic diseases. It is important that FNDC5/irisin regulation was reported in mouse liver, but its expression was not changed in skeletal muscle or adipose tissue. According to prominent studies, CAR is mainly expressed in the liver and intestine (3).

Generally, the metabolic function of irisin is known to be increasing energy expenditure and glucose hemostasis. Thus, it is possible that FNDC5 and irisin are increased in response to endurance training and aerobic training, which can be noticed by the increase in oxidation and metabolism (22). Another beneficial exerciseinduced effect of FNDC5/irisin is that irisin could inhibit the synthesis of hepatic cholesterol via AMPK-dependent inhibition of SREBP2 and its downstream target genes. Irisin increases the phosphorylation of AMPK $\alpha$ in hepatocytes. Blockage of irisin-induced AMPK activation by compound $\mathrm{C}$ or knockdown of AMPK $\alpha 1$ reverses the suppressive effects of irisin on: 1) hepatic cholesterol synthesis; 2) mRNA expression of SREBP2 and its downstream target genes crucial for cholesterol synthesis; and 3) nuclear translocalization of SREBP2. Irisin inhibits the synthesis of hepatic cholesterol via the mechanism dependent of AMPK-SREBP2 signaling. These findings may give insight in the treatment of diseases related to hypercholesterolemia, such as atherosclerosis (51).

In summary, previous research support that training is an influential factor in the expression 
of genes involved in energy metabolism (20). With respect to different physical trainings, metabolic adaptations originating from aerobic training have attracted the most attention; however, the potential value of resistance training in health and fitness have also been recognized. The significance of such training is that resistance training can, through the medium of a similar cell path, lead to adaptations similar to those that occur in endurance training while maintaining or increasing total muscle mass (25).

\section{CONCLUSION}

As it was shown here, resistance and endurance training can influence the liver FNDC5 gene expression similarly, considerably increasing liver FNDC5 mRNA in comparison to the control group. Thus, it is apparent that performing either aerobic or resistance training can stimulate the process of browning of white fat and improve the effects of fat amassment on the liver tissue. However, further research needs to be conducted so that the mechanisms involved in the effectiveness of different trainings on gene expression and irisin co-activator are fully comprehended and more helpful and efficient training protocols are put forward.

\section{APPLICABLE REMARKS}

- This study supports that both aerobic and resistance training can similarly increase FNDC5 gene expression in the liver tissue, which could be an explanation for the way they play a role in the process of browning of white fat, therefore reducing the fat in the liver tissue and imnroving the condition.

\section{REFERENCES}

1. Shimizu N, Maruyama T, Yoshikawa N, Matsumiya R, Ma Y, Ito N, et al. A muscle-liver-fat signaling axis is essential for central control of adaptive adipose remodeling. Nat Commun. 2015; 6(6693): 1-14.

2. Choi ES, Kim MK, Song MK, Kim JM, Kim ES, Chung WJ, et al. Association between Serum Irisin Levels and Non Alcoholic Fatty Liver Disease in Health Screen Examinees. PLoS ONE. 2014; 9(10): 110680- 7.

3. Mo L, Shen J, Liu Q, Zhang Y, Kuang J, Pu S, et al. Irisin Is Regulated by CAR in Liver and Is a Mediator of Hepatic Glucose and Lipid Metabolism. Mol Endocrinol. 2016; 30(5): 533-42.

4. Khodadadi H, Rajabi H, Seyyed Reza Attarzadeh S R, Abbasian S. The Effect of High Intensity Interval Training (HIIT) and Pilates on Levels of Irisin and Insulin Resistance in Overweight Women. Iranian Journal of Endocrinology and Metabolism 2014; 16(3): 190-6.

5. Arias-Loste MT, Ranchal I, Romero-Gómez M, Crespo J. Irisin, a Link among Fatty Liver Disease, Physical Inactivity and Insulin Resistance. International Journal of Molecular Sciences. 2014; 15(12):2316378.

6. Panati K, Suneetha Y, Narala VR. Irisin/FNDC5 - An updated review. European Review for Medical and Pharmacological Sciences. 2016; 20(4): 689-97.

7. Aghamohammadi M, Habibi A, Ranjbar R. The Effect of Selective Aerobic Training on Serum Irisin Levels and Insulin Resistance Index in Women with Type 2 Diabetes. Arak University of Medical Sciences Journal. 2016; $18(11): 1-9$

8. Aydin S. Three new players in energy regulation: Preptin, adropinand irisin. Peptides. 2014; 56: 94-110.

9. Erickson HP. Irisin and FNDC5 in retrospect: An exercise hormone or a transmembrane receptor?. Adipocyte. 2013; 2(4): 289-93.

10. Barja-Fernández S, Folgueira C, Castelao C, Al-Massadi O, Bravo SB, Garcia-Caballero T, et al. FNDC5 is produced in the stomach and associated to body composition. Scientific Reports. 2016;6:23067.

11. Schumacher MA, Chinnam N, Ohashi T, Shah RS, Erickson HP. The Structure of Irisin Reveals a Novel Intersubunit-Sheet Fibronectin Type III (FNIII) Dimer. Journal of Biologycal Chemisty. 2013; 288(47):3373844.

12. Srivastava N. ATP binding cassette transporter A1 - key roles in cellular lipid transport and atherosclerosis. Molecular and Cellular Biochemistry. 2002;237: 155-64.

13. Lecker SH, Zavin A, Cao P, Arena R, Allsup KM, Daniels KM, et al. Expression of the Irisin Precursor FNDC5 in Skeletal Muscle Correlates With Aerobic Exercise Performance in Patients With Heart Failure. Circ Heart Fail. 2012; 5(6): 812- 8.

14. Samy DM, Ismail CA, Nassra RA. Circulating Irisin Concentrations in Rat Models of Thyroid DysfunctionEffect of Exercise. Metabolism Clinical AND Experimental. 2015; 64(7): 804-13. 
15. Arias-Loste MT, Ranchal I, Romero-Gómez M, Crespo J. Irisin, a Link among Fatty Liver Disease, Physical Inactivity and Insulin Resistance. Int. J. Mol. Sci. 2014; 15: 23163-78.

16. Zhang HJ, Zhang XF, Ma ZM, Pan LL, Chen Z, Han HW, Han CK, Zhuang XJ, Lu Y, Li XJ, et al. Irisin is inversely associated with intrahepatic triglyceride contents in obese adults. J. Hepatol. 2013; 59: 557-62.

17. Norheim F, Langleite TM, Hjorth M, Holen T, Kielland A, Stadheim HK, et al. The effects of acute and chronic exercise on PGC-1a, irisin and browning of subcutaneous adipose tissue in humans. 2014; 281(3): 739-49.

18. Miyamoto-Mikami E, Sato K, Kurihara T, Hasegawa N, Fujie S, Fujita S, et al. Endurance Training-Induced Increase in Circulating Irisin Levels Is Associated with Reduction of Abdominal Visceral Fat in Middle-Aged and Older Adults. PLOS ONE. 2015; 10(3): e0120354.

19. Lui J, Cui XY, Yang YQ, Gao W, Sun L, Dong YC, et al. Effects of high-intensity treadmill training on timeliness and plasticity expression of irisin in mice. EuropeanReviewforMedical andPharmacological Sciences. 2015; 19(12): 2168-73.

20. Boström P, Wu J, Jedrychowski MP, Korde A, Ye L, Lo JC, et al. A PGC1 $\alpha$-dependent myokine that drives browning of white fat and thermogenesis. Nature. 2012; 481(7382):463-68.

21. Lally JS, Ford RJ, Johar J, Crane JD, Kemp BE, Steinberg GR. Skeletal muscle AMPK is essential for the maintenance of FNDC5 expression. Physiological Reports. 2015; 3(5): e12343.

22. Pekkala S, Wiklund PK, Hulmi JJ, Ahtiainen JP, Horttanainen M, Pollanen E, et al. Are skeletal muscleFNDC5gene expression and irisin release regulated by exercise and related to health?. J Physiol. 2013; 591(21): 5393-400.

23. Disanzo BL, You T. Effects of exercise training on indicators of adipose tissue angiogenesis and hypoxia in obese rats. Metabolism Clinical and Experimental. 2014; 63(4):452-5.

24. Matinhomaee H, Ziaolhagh SJ, Azarbayjani MA, Piri M. Effects of Boldenone consumption and resistance exercise on hepatocyte morphologic damages in male wistar rats. European Journal of Experimental Biology. 2014; 4(2): 211-4.

25. Reisi J, Rajabi H, Ghaedi K, Marandi SM, Asadysamani Z, Kazeminasab F. Effect of eight weeks' resistance training on plasma irisin protein level and muscle FNDC5 and adipose tissue UCP1 genes expression in male rats. Exercise phisiology. 2015; 7(28):117- 30.

26. Ghanbari-Niaki A, Ghanbari-Abarghooi S, Rahbarizadeh F, Zare-Kookandeh N, Gholizadeh M, Roudbari F, et al. Heart ABCA1 and PPAR- $\alpha$ Genes Expression Responses in Male rats: Effects of High Intensity Treadmill Running Training and Aqueous Extraction of Black Crataegus-Pentaegyna. Research in Cardiovascular Medicine. 2013; 1(5):153-9.

27. Livak KJ, Schmittgen TD. Analysis of Relative Gene Expression Data Using Real-Time Quantitative PCR and the 2- $\Delta \Delta$ Ct Method. Methods. 2001; 25(4): 402-8.

28. Liang $\mathrm{W}$ et al. Coordinated and interactive expression of genes of lipid metabolism and inflammation in adipose tissue and liver during metabolic overload. PLoS One. 2013;8(9):e75290.

29. Kawano Y, Cohen DE. Mechanisms of hepatic triglyceride accumulation in non-alcoholic fatty liver disease. J Gastroenterol. 2013;48(4):434-41.

30. Mavrogiannaki AN, Migdalis IN. Nonalcoholic fatty liver disease, diabetes mellitus and cardiovascular disease: newer data. Int J Endocrinol. 2013;2013:450639.

31. Kouis P, Pampaka D, Panayiotou AG. ADIPOSE TISSUE, METABOLIC SYNDROME, AND NONALCOHOLIC FATTY LIVER DISEASE - A SHORT REVIEW. EMJ Hepatol. 2014; 1: 62-70.

32. Huh JY, PanagiotouG, Mougios V, Brinkoetter M, Vamvini MT, Schneider BE, et al. FNDC5 and irisin in humans: I. Predictors of circulating concentrations in serum and plasma and II. mRNA expression and circulating concentrations in response to weight loss and exercise. Metabolism. 2012; 61:1725-38.

33. Komolka K, Albrecht E, Schering L, Brenmoehl J, Hoeflich A, Maak S. Locus characterization and gene expression of bovine FNDC5: Is the myokine irisin relevant in cattle? PloS One. 2014; 9: e88060.

34. Aydin S, Kuloglu T, Aydin S, Eren MN, Celik A, Yilmaz M, et al. Cardiac, skeletal muscle and serum irisin responses to with or without water exercise in young and old male rats: Cardiac muscle produces more irisin than skeletal muscle. Peptides. 2014;52:68-73.

35. Aydin S, Kuloglu T, Aydin S, Kalayci M, Yilmaz M, Cakmak T, et al. A comprehensive immunohistochemical examination of the distribution of the fat-burning protein irisin in biological tissues. Peptides. 2014;61:130-6.

36. Moon HS, Dincer F, Mantzoros CS. Pharmacological concen-trations of irisin increase cell proliferation without influenc-ing markers of neurite outgrowth and synaptogenesis in mouse H19-7 hippocampal cell lines. Metabolism. 2013;62(8):1131-6.

37. Polyzos SA, Kountouras J, Shields K, Mantzoros CS. Irisin: A renaissance in metabolism? Metabolism. 2013;62:1037-44.

Rashidlamir, A., Hoseinzadeh, M., Zeiaddini Dashtkhaki, L. (2017). Ann Appl Sport Sci, 5(2): 51-60. 
38. Cali AM, Zern TL, Taksali SE, de Oliveira AM, Dufour S, Otvos JD, Caprio S. Intrahepatic fat accumulation and alterations in lipoprotein composition in obese adolescents: A perfect proatherogenic state. Diabetes Care. 2007; 30: 3093-8.

39. Wong VW, Chu WC, Wong GL, Chan RS, Chim AM, Ong A, Yeung DK, Yiu KK, Chu SH, Woo J, et al. Prevalence of non-alcoholic fatty liverdisease and advanced fibrosis in Hong Kong Chinese: A population study using proton-magnetic resonance spectroscopy and transient elastography. Gut. 2012; 61: 409-5.

40. Badimon L, Vilahur G. LDL-cholesterolversusHDL-cholesterol in the atherosclerotic plaque: Inflammatory resolutionversusthrombotic chaos. Ann. N. Y. Acad. Sci. 2012; 1254: 18-32.

41. Holzer M, Birner-Gruenberger R, Stojakovic T, El-Gamal D, Binder V, Wadsack C, Heinemann A, Marsche G. Uremia alters HDL composition and function. J. Am. Soc. Nephrol. 2011; 22: 1631-41.

42. Hondares E, Rosell M, Diaz-Delfin J, Olmos Y, Monsalve M, Iglesias R, Villarroya F, Giralt M. Peroxisome proliferator-activated receptor alpha (PPARalpha) induces PPARgamma coactivator 1alpha (PGC-1alpha) gene expression and contributes to thermogenic activation of brown fat: Involvement of PRDM16. J. Biol. Chem. 2011, 286, 43112-22.

43. Xu J, Lloyd DJ, Hale C, Stanislaus S, Chen M, Sivits G, et al. Fibroblast growth factor 21 reverses hepatic steatosis, increases energy expenditure, and improves insulin sensitivity in diet-induced obese mice. Diabetes. 2009; 58: 250-9.

44. Fain JN, Company JM, Booth FW, Laughlin MH, Padilla J, Jenkins NT, et al. Exercise training does not increase muscle FNDC5 protein or mRNA expression in pigs. Metabolism. 2013; 62(10): 1503- 11.

45. Trayhurn P, Temple NJ, Van Aerde J. Evidence from immunoblotting studies on uncoupling protein that brown adipose tissue is not present in the domestic pig. Can J Physiol Pharmacol. 1989; 67(12):1480-5.

46. Berg F, Gustafson U, Andersson L. The uncoupling protein 1gene (UCP1) is disrupted in the pig lineage: a genetic explanation for poor thermoregulation in piglets. PLoS Genet. 2006; 2(8): 1178-81.

47. Cypess AM et al. Identification and importance of brown adipose tissue in adult humans. N Engl J Med. 2009; 360(15): 1509-17.

48. Yilmaz Y, Ones T, Purnak T, Ozguven S, Kurt R, Atug O, et al. Association between the presence of brown adipose tissue and non-alcoholic fatty liver disease in adult humans. Aliment Pharmacol Ther. 2011; 34(3): 31823.

49. Russell AP, Feilchenfeldt J, Schreiber S, Praz M, Crettenand A, Gobelet C, et al. Endurance Training in Humans Leads to Fiber Type-Specific Increases in Levels of Peroxisome Proliferator-Activated Receptor-Coactivator-1 and Peroxisome Proliferator- $\gamma$ Activated Receptor- $\alpha$ in Skeletal Muscle. Diabetes. 2003; 52(12): $2874-81$.

50. Butcher LR, Thomas A, Backx K, Roberts A, Webb R, Morris K. Low-Intensity Exercise Exerts Beneficial Effects on Plasma Lipids via PPAR (gamma). Medicine \& Science in Sports \& Exercise. 2008; 40(7): $1263-70$.

51. Tang H, Yu R, Liu S, Huwatibieke B, Li Z, Zhang W. Irisin Inhibits Hepatic Cholesterol Synthesis via AMPKSREBP2 Signaling. EbioMedicine. 2016; 6: 139-48. 